Check for updates

Cite this: Chem. Sci., 2019, 10, 6715

¿ All publication charges for this article have been paid for by the Royal Society of Chemistry

\section{Consecutive HDDA and TDDA reactions of silicon- tethered tetraynes for the synthesis of dibenzosilole-fused polycyclic compounds and their unique reactivity $\dagger$}

\author{
Akihito Mitake, ${ }^{a}$ Rikako Nagai, ${ }^{a}$ Ayato Sekine, ${ }^{a}$ Hideaki Takano, ${ }^{a}$ Natsuhiko Sugimura, ${ }^{b}$ \\ Kyalo Stephen Kanyiva (D) ${ }^{c}$ and Takanori Shibata (D) *a
}

Received 25th February 2019

Accepted 25th May 2019

DOI: $10.1039 / \mathrm{c} 9 \mathrm{sc} 00960 \mathrm{~d}$

rsc.li/chemical-science

\begin{abstract}
Silicon-tethered tetraynes possessing a 1,3-diyne moiety underwent consecutive hexadehydro- and tetradehydro-Diels-Alder reactions to give a series of fused polycyclic aromatic compounds containing a dibenzosilole skeleton. The benzene ring in the product acted as a 1,3-diene and reacted with the active alkyne as well as oxygen to provide [4 +2$]$ cycloadducts.
\end{abstract}

While the Diels-Alder reaction is a [4+2] cycloaddition of a 1,3diene and an alkene, the dehydro-Diels-Alder (DDA) reaction involves an alkyne moiety(ies) in the substrate(s). ${ }^{1}$ For example, the tetradehydro-Diels-Alder (TDDA) reaction of a 1,3-enyne and an alkyne gives a substituted benzene ring with perfect atom-economy via a strained cyclic allene intermediate along with a 1,5-hydrogen shift. In particular, the intramolecular TDDA reaction of arylalkynes, where part of the arene acts as an ene moiety, is attractive, because fused polycyclic aromatic compounds can be prepared in one pot. Saá is a pioneer in the synthetic use of TDDA and comprehensively studied an intramolecular reaction of diarylacetylene and alkyne; ${ }^{2}$ the reaction of ynamides gave carbazole derivatives (Scheme 1a). ${ }^{3}$ Our group also reported an intramolecular TDDA reaction for the synthesis of binaphthyl compounds. ${ }^{4}$ Recently, we developed the consecutive intramolecular TDDA reaction of sulfur-tethered tetraynes for the preparation of axially chiral bis(benzothiophene) derivatives and further upgraded this transformation to an enantioselective synthesis by a chiral metal-catalyzed reaction (Scheme 1b). ${ }^{5}$

Against this background, we next examined the thermal reaction using silicon analogue $\mathbf{1 a}^{\mathbf{6}}$ in hot toluene for the preparation of an axially chiral bis(benzosilole) derivative. Unexpectedly, we obtained dibenzosilole-fused heptacyclic compound $\mathbf{2 a}$, the structure of which was finally decided upon based on X-ray analysis (Scheme 2). We considered that consecutive intramolecular DDA reactions gave polycyclic compounds. The first step is a hexadehydro-Diels-Alder (HDDA) reaction of 1,3-diyne and alkyne to give benzosilole-fused benzyne and the second step is a TDDA reaction with the remaining arylalkyne moiety. Since the first report of the HDDA reaction, ${ }^{7 a}$ Hoye reported various reagents for trapping of the reactive benzyne intermediates. ${ }^{7 b-v}$ Recently, consecutive HDDA reactions were developed for the one-pot synthesis of fused polycyclic compounds. ${ }^{7 w}$ In contrast, we demonstrate here the first example of consecutive HDDA and TDDA reactions as well as a HDDA reaction along with $[2+2+2+2]$ cycloaddition. We further discuss the unique reactivity of the benzene moiety of the silicon-containing polycyclic compound.

a) Intramolecular TDDA reaction of ynamides (ref. 3)

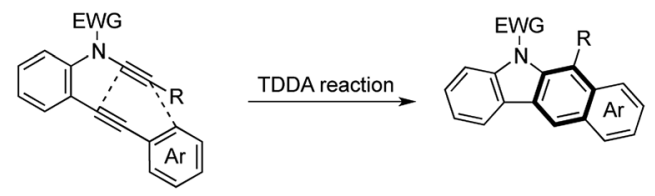

b) Consecutive intramolecular TDDA reactions of alkynyl sulfides (ref. 5)
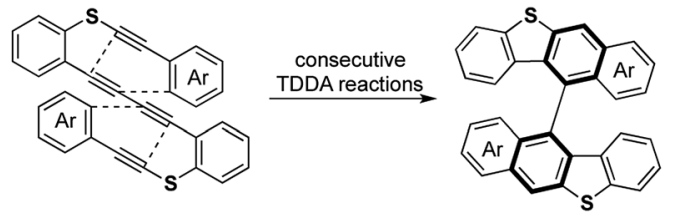

Scheme 1 Intramolecular TDDA reactions for the synthesis of dibenzoheteroles.

${ }^{c}$ Global Center for Science and Engineering, School of Advanced Science and Engineering, Waseda University, Shinjuku, Tokyo 169-8555, Japan

$\dagger$ Electronic supplementary information (ESI) available. CCDC 1826048, 1826049, 1890968 and 1830394. For ESI and crystallographic data in CIF or other electronic format see DOI: $10.1039 / \mathrm{c} 9 \mathrm{sc00960d}$ 


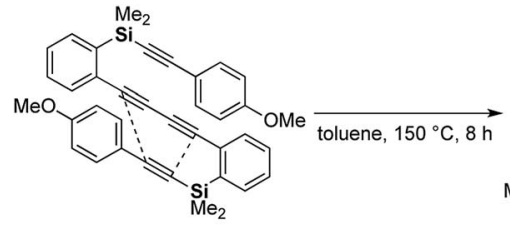

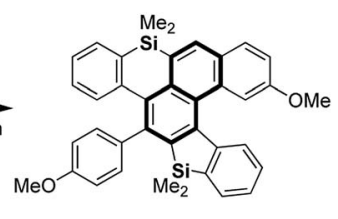

$1 \mathrm{a}$

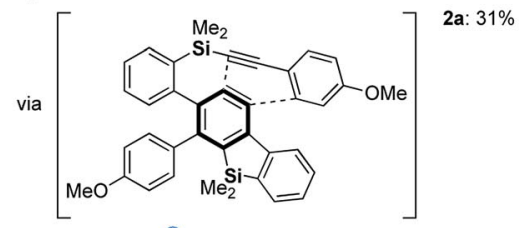

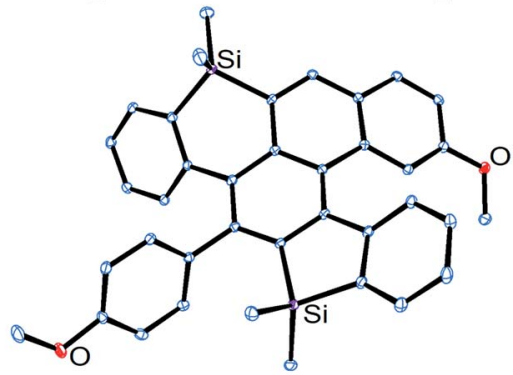

Ortep diagram of cycloadduct $2 a$ (Thermal ellipsoids shown at $50 \%$ probability ${ }^{[8]}$ )

Scheme 2 Consecutive HDDA and TDDA reactions of silicon-tethered tetrayne 1a possessing a 1,3-diyne moiety.

We chose 1,4-bis(2-(dimethyl-(2-(4-methoxyphenyl)ethynyl)silyl)phenyl)buta-1,3-diyne (1a) as a model substrate and screened the thermal conditions for the consecutive HDDA and TDDA reactions (Table 1). When dibutyl ether was used, tetrayne 1a was completely consumed within $8 \mathrm{~h}$ and dibenzosilole-fused cycloadduct 2a was obtained in moderate NMR yield (entry 1). While benzonitrile realized a yield comparable to that with the etherate solvent, propionitrile gave the best yield of

Table 1 Screening of thermal conditions for the consecutive DDA reactions $^{a}$
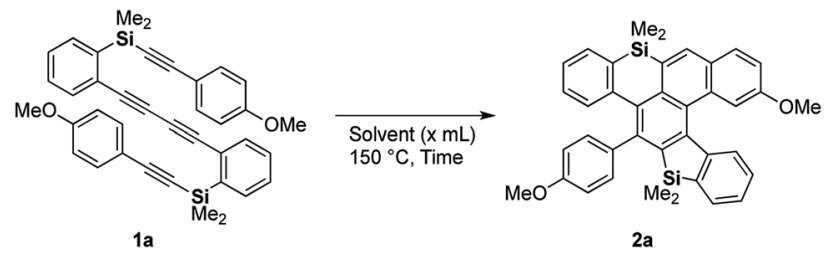

\begin{tabular}{llll}
\hline Entry & Solvent $(x \mathrm{~mL})$ & Time $(\mathrm{h})$ & NMR yield $^{b}(\%)$ \\
\hline 1 & Dibutyl ether (5) & 8 & 56 \\
2 & PhCN (5) & 4 & 52 \\
3 & $\mathrm{CH}_{3} \mathrm{CH}_{2} \mathrm{CN} \mathrm{(5)}$ & 48 & 72 \\
4 & $\mathrm{CH}_{3} \mathrm{CH}_{2} \mathrm{CN} \mathrm{(1.7)}$ & 48 & 41 \\
5 & $\mathrm{CH}_{3} \mathrm{CH}_{2} \mathrm{CN}(15)$ & 48 & 83 \\
$6^{c}$ & $\mathrm{CH}_{3} \mathrm{CH}_{2} \mathrm{CN}(3)$ & 1 & 27
\end{tabular}

${ }^{a}$ The reaction was conducted on a $0.05 \mathrm{mmol}$ scale. ${ }^{b}$ Yields were determined by NMR using 1,1,2,2-tetrachloroethane as an internal standard. ${ }^{c}$ The reaction was conducted on a $0.03 \mathrm{mmol}$ scale under microwave irradiation.
Table 2 Substrate scope of tetrayne $1 a^{a}$
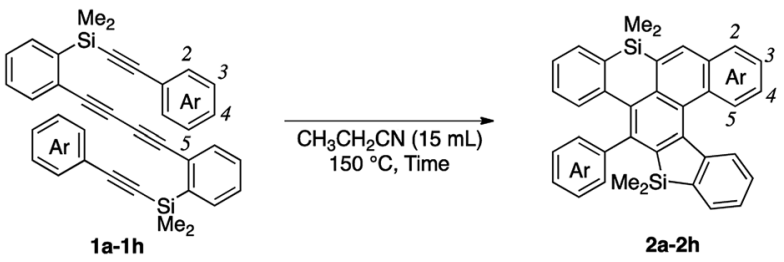

\begin{tabular}{llll}
\hline Entry & Ar & Time (h) & Yield (\%) \\
\hline 1 & $4-\mathrm{MeOC}_{6} \mathrm{H}_{4}$ & 40 & $81(\mathbf{2 a})$ \\
2 & $\mathrm{C}_{6} \mathrm{H}_{5}$ & 48 & $55(2 \mathbf{b})$ \\
3 & $4-\mathrm{FC}_{6} \mathrm{H}_{4}$ & 48 & $51(2 \mathbf{c})$ \\
4 & $3,5-\left(\mathrm{MeO}_{2} \mathrm{C}_{6} \mathrm{H}_{3}\right.$ & 24 & $56(\mathbf{2 d})$ \\
5 & $2-\mathrm{MeOC}_{6} \mathrm{H}_{4}$ & 40 & $52(\mathbf{2 e})$ \\
6 & $4-\mathrm{PhC}_{6} \mathrm{H}_{4}$ & 24 & $43(53)^{b}(\mathbf{2 f})$ \\
7 & 1-Naphthyl & 24 & $53(2 \mathbf{g})$ \\
8 & 2-Benzothienyl & 24 & $51(\mathbf{2 h})$
\end{tabular}

${ }^{a}$ Tetrayne $(0.05 \mathrm{mmol})$ and propionitrile $(15 \mathrm{ml})$ were used and the product was purified by preparative TLC. ${ }^{b}$ Yield in parenthesis was determined by NMR using 1,1,2,2-tetrachloroethane as an internal standard.

$72 \%$ with a longer reaction time (entries 2 and 3). After we investigated the concentration effect, the yield was improved to $83 \%$ under dilute conditions, but with a prolonged reaction time (entries 3-5). Tetrayne 1a was completely consumed within $1 \mathrm{~h}$ under microwave irradiation, but the reaction became messy, and the yield of 2a was low (entry 6). We determined that entry 5 represented the best conditions.

We next examined the substrate scope of aryl groups on the alkyne termini in propionitrile at $150{ }^{\circ} \mathrm{C}$ (Table 2). Compound 2a was obtained in $81 \%$ isolated yield. Phenyl- and 4-fluorophenyl-substituted tetraynes $\mathbf{1 b}$ and $\mathbf{1 c}$ were transformed into the corresponding heptacyclic compounds $\mathbf{2 b}$ and $2 \mathbf{c}$ in moderate yields. Electron-rich and ortho-substituted arenes could also be used and cycloadducts $2 \mathbf{d}$ and $2 \mathbf{e}^{\mathbf{9}}$ were obtained in moderate yields. In the case of 4-biphenyl-substituted product $2 \mathbf{f}$, the yield was low because it was difficult to isolate due to its high crystallinity. The reactions of 1-naphthyl and 2benzothiophenyl-substituted tetraynes $\mathbf{1 g}$ and $\mathbf{1 h}$ also proceeded to give octacyclic cycloadduct $\mathbf{2 g}^{\mathbf{9}}$ and dihetero[6] helicene $\mathbf{2 h}$ consisting of dibenzothiophene and dibenzosilole, respectively.
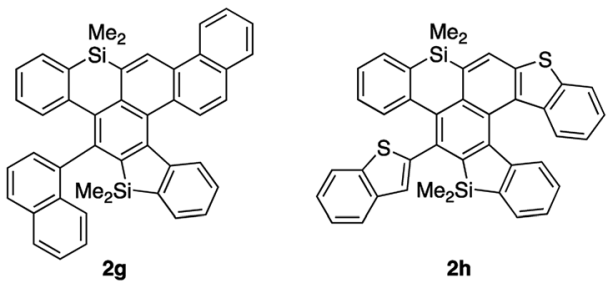

While the reaction of 2-naphthyl-substituted tetrayne $\mathbf{1 i}$ possibly affords two regioisomers $2 \mathbf{i}$ and $2 \mathbf{i}^{\prime}$, sila[6] helicene $\mathbf{2 i}$ was the only cycloadduct detected, probably due to the higher reactivity of the $\alpha$-position of the naphthyl group (Scheme 3). 


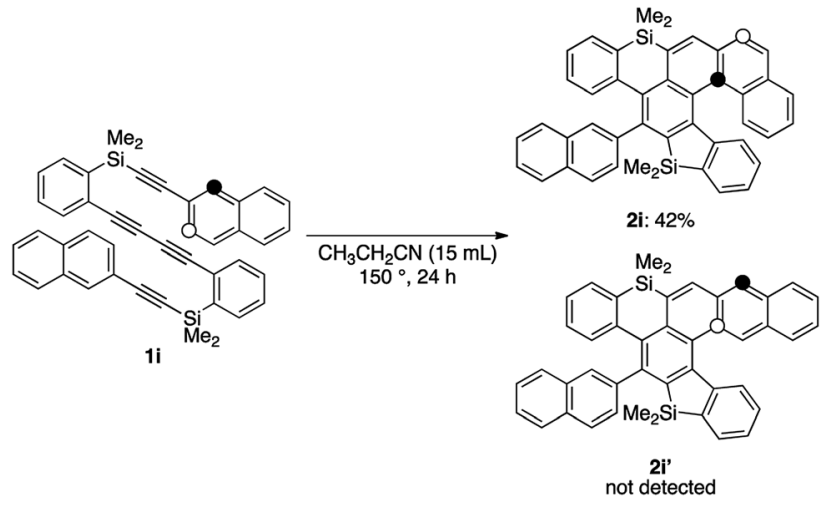

Scheme 3 Regioselective reaction of tetrayne $1 \mathrm{i}$.
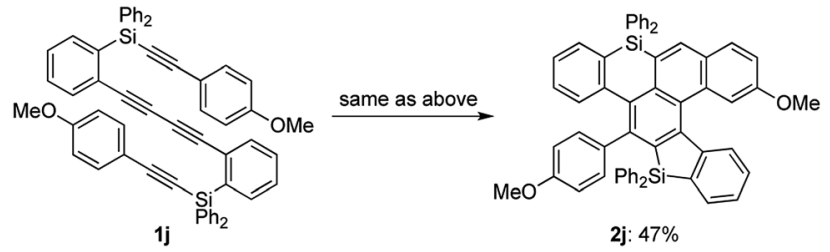

Scheme 4 Reaction of diphenylsilyl-tethered tetrayne $\mathbf{1 j}$.

Diphenylsilyl-tethered tetrayne $\mathbf{1} \mathbf{j}$ was also transformed into the corresponding cycloadduct $2 \mathbf{j}$ (Scheme 4).

We conducted mechanism study in order to figure out why silicon-tethered tetraynes underwent the HDDA reaction, not the TDDA reaction. As shown by the results of DFT calculations using triyne $\mathbf{A}$ as a model substrate, the activation energy of both HDDA and TDDA reactions via diradical intermediates ${ }^{7 k}$ was large (54.3 and $49.0 \mathrm{kcal} \mathrm{mol}^{-1}$, respectively). In contrast,

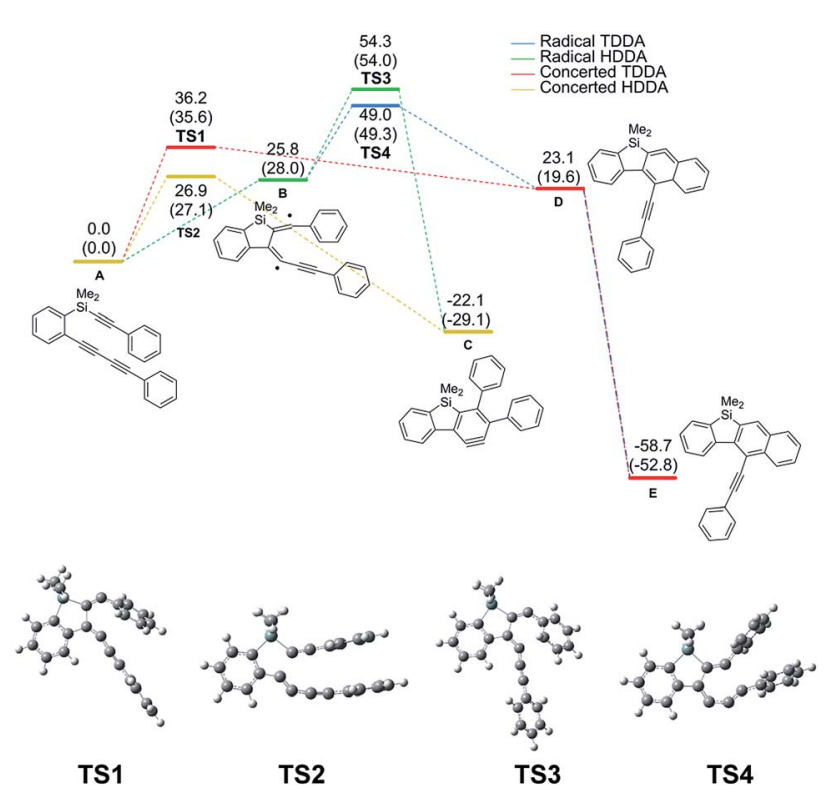

Fig. 1 Relative Gibbs free energy $(\Delta G)$ diagram of Si-tethered triyne A at $423.15 \mathrm{~K}$ ( $\mathrm{kcal} \mathrm{mol}^{-1}$ ). Relative electronic energy $(\Delta E)$ is in parentheses.
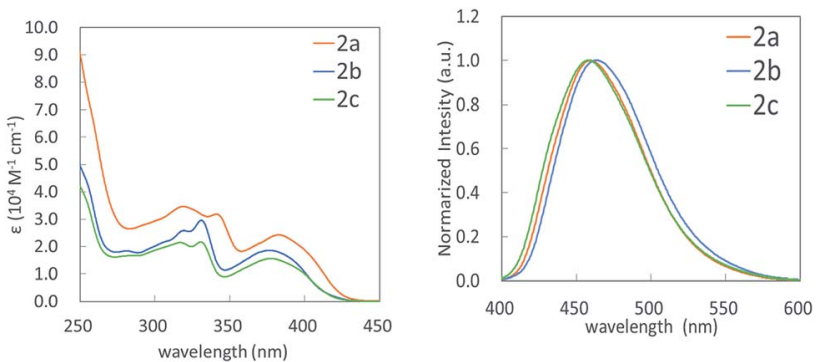

Fig. 2 UV-vis (left) and fluorescence spectra (right) of 2a-2c.

the concerted pathway showed smaller activation energy; moreover, the concerted HDDA reaction $\left(27.1 \mathrm{kcal} \mathrm{mol}^{-1}\right)$ was clearly more favorable than the concerted TDDA reaction (35.6 kcal mol ${ }^{-1}$ ) (Fig. 1). ${ }^{10}$

In all of the entries above, some by-products were always formed. Among them, the major isolated by-products were cyclooctatetraene derivatives. The structure of the by-product in the reaction of 1a was finally determined to be saddle-shaped compound 3a by X-ray crystallographic analysis (Scheme 5 below). We considered the mechanism to be dimerization of in situ-generated benzosilole-fused benzyne (Scheme 5 above). To the best of our knowledge, this is the first example of the thermal $[2+2+2+2]$ cycloaddition of alkynes for the construction of an eight-membered ring system. ${ }^{11}$ Therefore, we further examined the reaction conditions. ${ }^{12}$ As a result, $3 \mathbf{b}$ was obtained in moderate yield as a major product under more concentrated conditions in chlorobenzene (Scheme 6). ${ }^{13}$
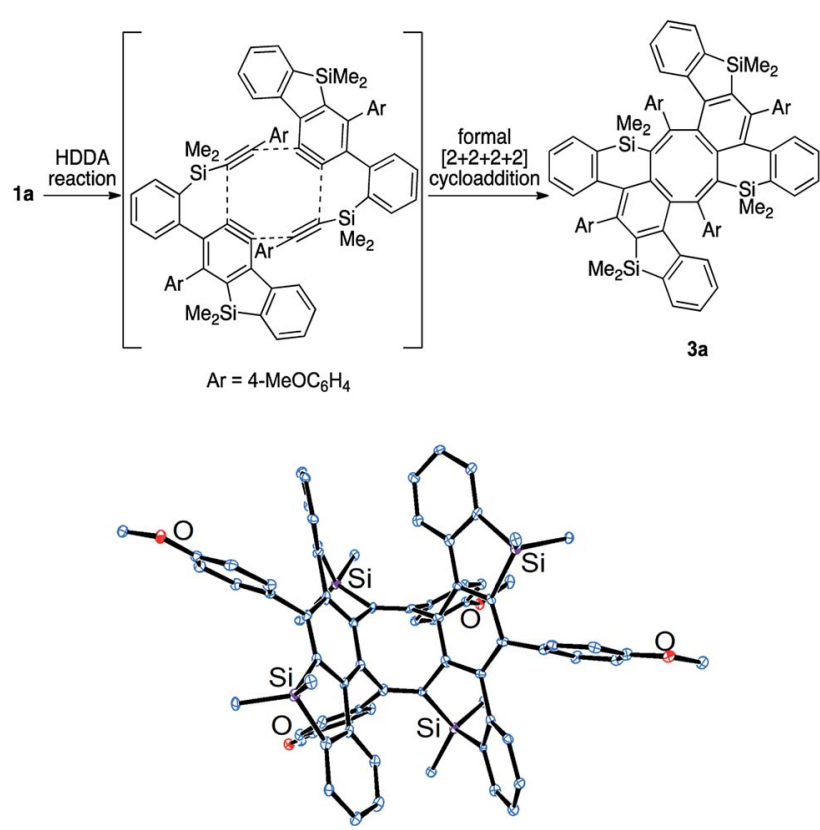

Ortep diagram of cycloadduct $3 \mathrm{a}$

(Thermal ellipsoids shown at $50 \%$ probability ${ }^{[8]}$ )

Scheme 5 Proposed mechanism for the formation of saddle-shaped compound 3 a. 


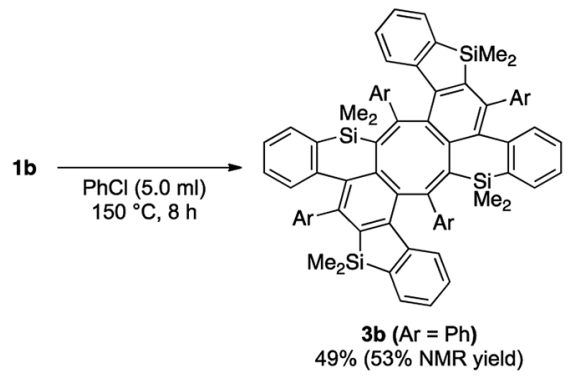

Scheme 6 Formal [2+2+2+2] cycloaddition of two benzynes and two alkynes.

Table 3 Photophysical properties of $2 a-2 c^{a}$

\begin{tabular}{llll}
\hline & $\begin{array}{l}\lambda_{\max (\mathrm{abs})}{ }^{b}[\mathrm{~nm}], \\
\left(\varepsilon\left[\times 10^{4} \mathrm{~cm}^{-1} \mathrm{M}^{-1}\right]\right)\end{array}$ & $\begin{array}{l}\lambda_{\max (\mathrm{emi})}{ }^{c, d} \\
{[\mathrm{~nm}]}\end{array}$ & $\begin{array}{l}\Phi^{c, d} \\
(\text { solution, solid) }\end{array}$ \\
Comp. & & \\
2a & $319(3.5), 341(3.2), 383(2.4)$ & 459 & $0.15,0.22$ \\
2b & $319(2.6), 331(3.0), 376(1.9)$ & 463 & $0.14,0.21$ \\
2c & $317(2.1), 331(2.2), 377(1.6)$ & 458 & $0.14,0.16$
\end{tabular}

${ }^{a} \mathrm{UV}$-vis and fluorescence spectra of 2 were measured in $\mathrm{CH}_{2} \mathrm{Cl}_{2} \cdot{ }^{b}$ 2a 1.7 $\times 10^{-5} \mathrm{M} ; 2$ b $2.1 \times 10^{-5} \mathrm{M} ; 2$ c $3.0 \times 10^{-5} \mathrm{M}^{c}{ }^{c} 2 \mathrm{a} 1.7 \times 10^{-6} \mathrm{M} ; 2 \mathrm{~b} 2.1 \times$ $10^{-6} \mathrm{M} ; 2 \mathbf{c} 3.0 \times 10^{-6} \mathrm{M}^{d}{ }^{d}$ Excitation wavelength: $2 \mathbf{a} 383 \mathrm{~nm} ; 2 \mathbf{b} 376 \mathrm{~nm}$; 2c $377 \mathrm{~nm}$.

We measured UV-vis and fluorescence spectra of benzosilolefused polycyclic compounds $\mathbf{2 a - 2 c}$ in dichloromethane as well as their quantum yields in both dichloromethane solution and the solid state (Fig. 2 and Table 3). These compounds are fluorescent and their quantum yields in the solid state were higher than those in solution. Substitution at the 6- and 15positions did not affect their photophysical properties. The Stokes shifts were twice as much as those for simple sila[5] helicene, ${ }^{\mathbf{1 4}}$ probably due to the fused siline ring. The torsion angle of the silahelicene moiety of $2 \mathrm{a}$ was $27.0^{\circ}$, which was significantly larger than that of the simple sila[5]helicene $\left(17.6^{\circ}\right) \cdot{ }^{14}$

We further investigated the reactivity of the unique $\pi$-system containing silicon atoms. We first conducted the reaction of silahelicene 2a with excess amounts of in situ-generated benzyne as a reactive alkyne; consecutive $[4+2]$ cycloaddition proceeded at $30{ }^{\circ} \mathrm{C}$ for $3 \mathrm{~h}$ to give polycyclic compound 4 possessing two bridged systems, the structure of which was

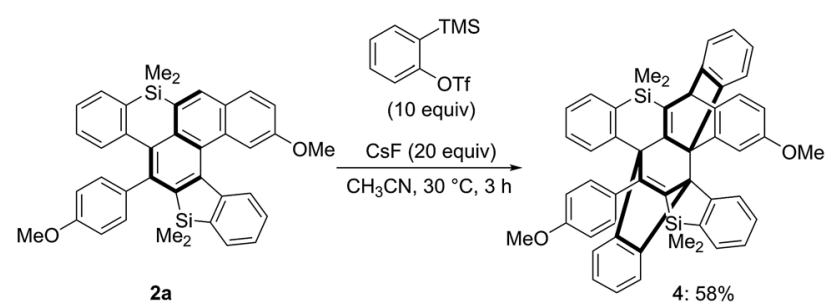

Scheme 7 Consecutive [4 +2$]$ cycloadditions of silahelicene $2 a$ with benzyne.

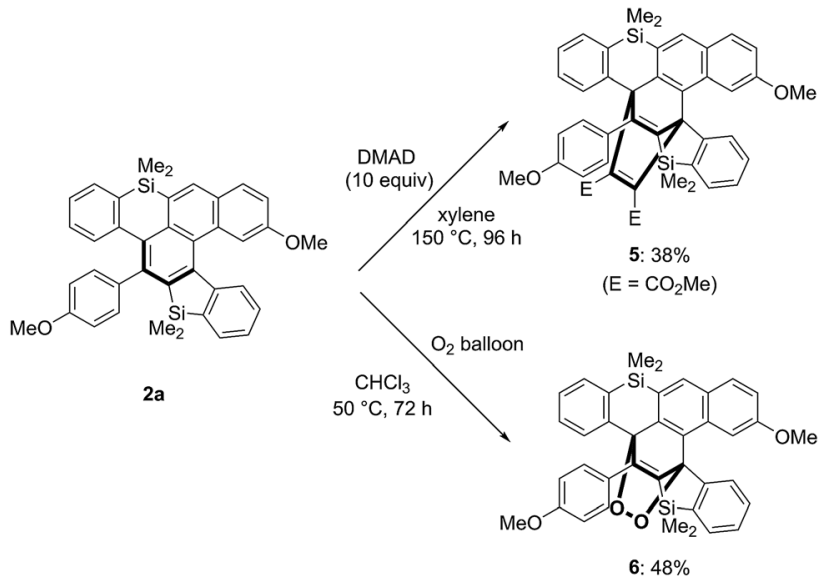

Scheme 8 Diels-Alder reactions of silahelicene $2 a$ with DMAD and oxygen.

confirmed by X-ray crystallographic analysis (Scheme 7).$^{15}$ Even when the amount of the benzyne precursor was decreased, a 1 : 1 cycloadduct could not be detected.

While the $[4+2]$ cycloaddition of $2 \mathbf{a}$ with dimethyl acetylenedicarboxylate (DMAD) required a high reaction temperature as well as a long reaction time, $1: 1$ cycloadduct 5 was the only detectable product (Scheme 8 above).$^{16}$ Interestingly, the reaction of 2a proceeded under an atmospheric pressure of oxygen at $50{ }^{\circ} \mathrm{C}$ to give cyclic peroxide 6 in moderate yield, the structure of which was also confirmed by X-ray crystallographic analysis (Scheme 8 below). ${ }^{17}$

To explain the high reactivity with oxygen, we calculated the values of NICS(0) for cycloadduct 2a and cyclic peroxide 6 (Table 4). ${ }^{18}$ The aromaticities of rings c and e of 2 a were relatively weak, because they are fused with the antiaromatic siline (ring b). Ring c gained aromaticity by $[4+2]$ cycloaddition with oxygen $(-3.30$ to -7.22$)$.

Table 4 NICS(0) of sila[5]helicene $2 a$ and $6^{a}$

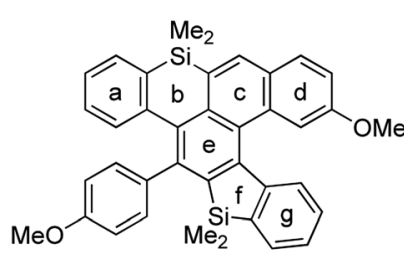

$2 a$

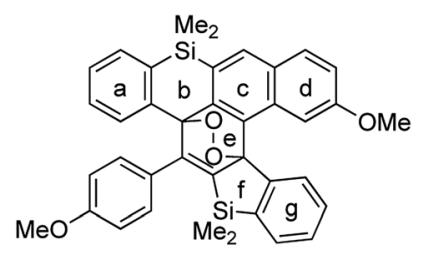

\begin{tabular}{lrr}
\hline Ring & \multicolumn{1}{c}{ 2a } & \multicolumn{1}{c}{6} \\
\hline a & -6.09 & -6.89 \\
$\mathrm{~b}$ & 4.50 & 3.68 \\
$\mathrm{c}$ & -3.30 & -7.22 \\
$\mathrm{~d}$ & -8.27 & -7.75 \\
$\mathrm{e}$ & -5.03 & 2.46 \\
$\mathrm{f}$ & 4.39 & 2.25 \\
$\mathrm{~g}$ & -4.85 & -6.22 \\
a GIAO B3LYP/6-31+G(d,p). & &
\end{tabular}


In conclusion, we have developed the first example of consecutive HDDA and TDDA reactions using silicon-tethered tetraynes possessing a 1,3-diyne moiety via benzosilole-fused benzynes. The obtained silicon-containing polycyclic aromatic compound acted as a diene and underwent $[4+2]$ cycloaddition with active alkynes. Notably, it could react with non-activated oxygen to give a cyclic peroxide.

\section{Conflicts of interest}

There are no conflicts to declare.

\section{Acknowledgements}

This work was partly executed under the cooperation of organization between Waseda University and JXTG Nippon Oil \& Energy Corporation. This work was also supported by a Grant-in-Aid for Scientific Research (C) (No. 19K05485) from JSPS.

\section{Notes and references}

1 For selected reviews of DDA reactions, see; (a) P. Wessig and G. Müller, Chem. Rev., 2008, 108, 2051-2063; (b) W. Li, L. Zhou and J. Zhang, Chem.-Eur. J., 2016, 22, 1558-1571.

2 (a) D. Rodríguez, A. Navarro, L. Castedo, D. Domínguez and C. Saá, Org. Lett., 2000, 2, 1497-1500; (b) D. Rodríguez, A. Navarro-Vázquez, L. Castedo, D. Domínguez and C. Saá, J. Org. Chem., 2003, 68, 1938-1946; (c) D. Rodríguez, M. F. Martínez-Esperón, A. NavarroVázquez, L. Castedo, D. Domínguez and C. Saá, J. Org. Chem., 2004, 69, 3842-3848.

3 (a) M. F. Martínez-Esperón, D. Rodríguez, L. Castedo and C. Saá, Org. Lett., 2005, 7, 2213-2216; (b) M. F. MartínezEsperón, D. Rodríguez, L. Castedo and C. Saá, Tetrahedron, 2006, 62, 3843-3855; (c) M. F. Martínez-Esperón, D. Rodríguez, L. Castedo and C. Saá, Tetrahedron, 2008, 64, 3674-3686.

4 T. Shibata, R. Fujiwara and D. Takano, Synlett, 2005, 20622066.

5 T. Shibata, A. Sekine, A. Mitake and K. S. Kanyiva, Angew. Chem., Int. Ed., 2018, 57, 15862-15865.

6 The silicon-tethered tetrayne 1a was subjected to the consecutive intramolecular $[2+2+2]$ cycloaddition with an ortho-phenylene-tethered diyne for the preparation of a helically chiral disila[7]helicene: T. Shibata, T. Uchiyama, Y. Yoshinami, S. Takayasu, K. Tsuchikama and K. Endo, Chem. Commun., 2012, 48, 1311-1313.

7 The first report of the HDDA reaction: (a) T. R. Hoye, B. Baire, D. Niu, P. H. Willoughby and B. P. Woods, Nature, 2012, 490, 208-212. Examples of the reaction of benzyne generated by HDDA with various trapping reagents: (b) D. Niu, P. H. Willoughby, T. R. Hoye, B. P. Wood and B. Baire, Nature, 2013, 501, 531-534; (c) D. Niu and T. R. Hoye, Nat. Chem., 2014, 6, 34-40; (d) T. R. Hoye, B. Baire and T. Wang, Chem. Sci., 2014, 5,
545-550; (e) D. Niu, T. Wang, B. P. Woods and T. R. Hoye, Org. Lett., 2014, 16, 254-257; $(f)$ J. Chen, B. Baire and T. R. Hoye, Heterocycles, 2014, 88, 1191-1200; $(g)$ P. H. Willoughby, D. Niu, T. Wang, M. K. Haj, C. J. Cramer and T. R. Hoye, J. Am. Chem. Soc., 2014, 136, 13657-13665; (h) V. D. Pogula, T. Wang and T. R. Hoye, Org. Lett., 2015, 17, 856-859; (i) Q. L. Nguyen, B. Baire and T. R. Hoye, Tetrahedron Lett., 2015, 56, 3265-3267; (j) J. Chen, V. Palani and T. R. Hoye, J. Am. Chem. Soc., 2016, 138, 4318-4321; ( $k$ ) T. Wang, D. Niu and T. R. Hoye, J. Am. Chem. Soc., 2016, 138, 7832-7835; (l) T. Wang and T. R. Hoye, J. Am. Chem. Soc., 2016, 138, 13870-13873; (m) J. Zhang, D. Niu, V. A. Brinker and T. R. Hoye, Org. Lett., 2016, 18, 5596-5599; (n) F. Xu, X. Xiao and T. R. Hoye, Org. Lett., 2016, 18, 5636-5639; (o) V. Palani, J. Chen and T. R. Hoye, Org. Lett., 2016, 18, 6312-6315; (p) S. P. Ross and T. R. Hoye, Nat. Chem., 2017, 9, 523-530; (q) S. P. Ross, B. Baire and T. R. Hoye, Org. Lett., 2017, 19, 5705-5708; (r) Y. Wang and T. R. Hoye, Org. Lett., 2018, 20, 88-91; $(s)$ S. P. Ross and T. R. Hoye, Org. Lett., 2018, 20, 100-103; $(t)$ J. Zhang, A. C. S. Page, V. Palani, J. Chen and T. R. Hoye, Org. Lett., 2018, 20, 5550-5553; (u) Y. Wang, L. Zheng and T. R. Hoye, Org. Lett., 2018, 20, 7145-7148; (v) S. Arora, V. Palani and T. R. Hoye, Org. Lett., 2018, 20, 8082-8085. Example of recent consecutive HDDA reactions: $(w) \mathrm{X}$. Xiao and T. R. Hoye, Nat. Chem., 2018, 10, 838-844.

8 CCDC 1826048 for 2a, CCDC 1826049 for 3a, CCDC 1890968 for 4, CCDC 1830394 for 6 contain the supplementary crystallographic data for this paper. $\dagger$

9 Compounds $2 \mathrm{e}$ and $2 \mathrm{~g}$ have axial chirality as well as helical chirality, and they were obtained as a diastereomeric mixture.

10 A tetrayne tethered by a methylene moiety in place of a dimethylsilyl one underwent consecutive intramolecular TDDA reactions to afford a bibenzo[b]fluorene derivative, which was shown in detail in the ESI. $\dagger$

11 Example of thermal $[2+2+2+2]$ cycloaddition of an alkene: (a) P. Camps, J. A. Fernández, S. Vázquez, M. Font-Bardia and X. Solans, Angew. Chem., Int. Ed., 2003, 42, 4049-4051. Example of metal-catalyzed $[2+2+2+2]$ cycloaddition of an alkyne: $(b)$ P. A. Wender, M. P. Croatt and B. Kühn, Organometallics, 2009, 28, 5841-5844; (c) D. J. Nasrallah and M. P. Croatt, Eur. J. Org. Chem., 2014, 18, 3767-3772. Example of photochemical $[2+2+2+2]$ cycloaddition of furan to $\beta$-naphthonitrile: $(d)$ T. Sugioka, C. Pac and H. Sakurai, Chem. Lett., 1972, 9, 791-792.

12 The table for the reaction condition screening is listed in ESI. $\dagger$

13 To improve the yield of formal $[2+2+2+2]$ cycloadduct, we tried to suppress the TDDA reaction of the second step. But the reaction of $n$-butyl-substituted tetrayne did not proceed at all. In the reaction of 2,6-dimethoxy-substituted tetrayne, the benzyne intermediate from the HDDA reaction was probably formed, but the formation of the [2 $+2+2+2$ ] cycloadduct could not be detected. 
14 M. Murai, R. Okada, A. Nishiyama and K. Takai, Org. Lett., 2016, 18, 4380-4383.

15 S. Toyota, A. Takau, Y. Hitaka, M. Oki and K. Wakamatsu, Bull. Chem. Soc. Jpn., 2005, 78, 2228-2234.

16 Y. Kim, Z. Zhu and T. M. Swager, J. Am. Chem. Soc., 2004, 126, 452.
17 H. Kotani, K. Ohkubo and S. Fukuzumi, J. Am. Chem. Soc., 2004, 126, 15999-16006.

18 P. v. R. Schleyer, C. Maerker, A. Dransfeld, H. Jiao and N. J. R. v. E. Hommes, J. Am. Chem. Soc., 1996, 118, 63176318. 